\title{
Supporting Information for "Efficient Training of the Memristive Deep Belief Net Immune to Non-Idealities of the Synaptic Devices"
}

Wei Wang ${ }^{1}$, Barak Hoffer ${ }^{1}$, Tzofnat Greenberg-Toledo ${ }^{1}$, Yang Li $^{1}$, Minhui Zou ${ }^{1}$, Eric Herbelin $^{1}$, Ronny Ronen ${ }^{1}$, Xiaoxin $\mathrm{Xu}^{1}$, Yulin Zhao ${ }^{1}$, Jianguo Yang ${ }^{1}$, and Shahar Kvatinsky $^{1}$

${ }^{1}$ Affiliation not available

January 4, 2022
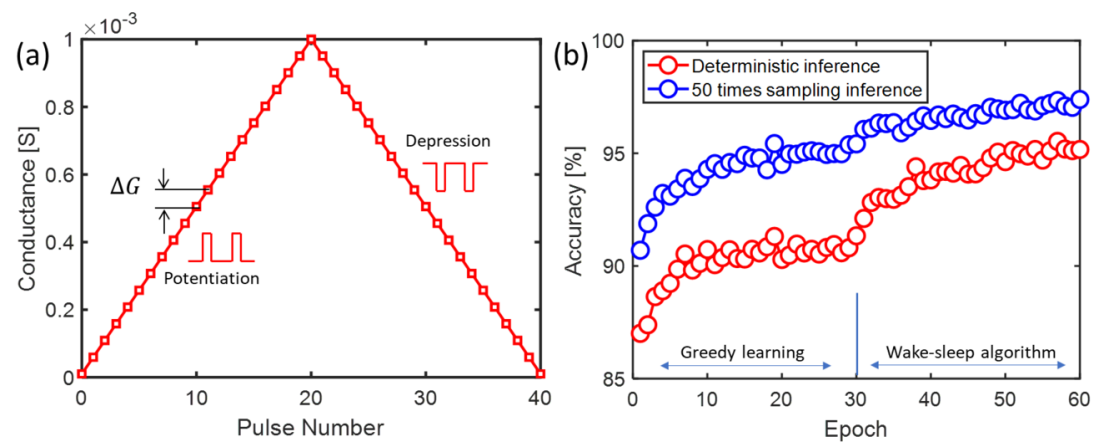

Figure S1. a) The memristive synaptic behavior with an ideally symmetric and linear weight update ability (constant $\Delta \mathrm{G}$ for identical pulses) but limited conductance levels $(N=20)$. b) Test accuracy for 10,000 images in the MNIST dataset obtained during the training of memristive DBN as a function of the training epoch $\left(\mathrm{CD}_{\mathrm{th}}=64\right)$. 

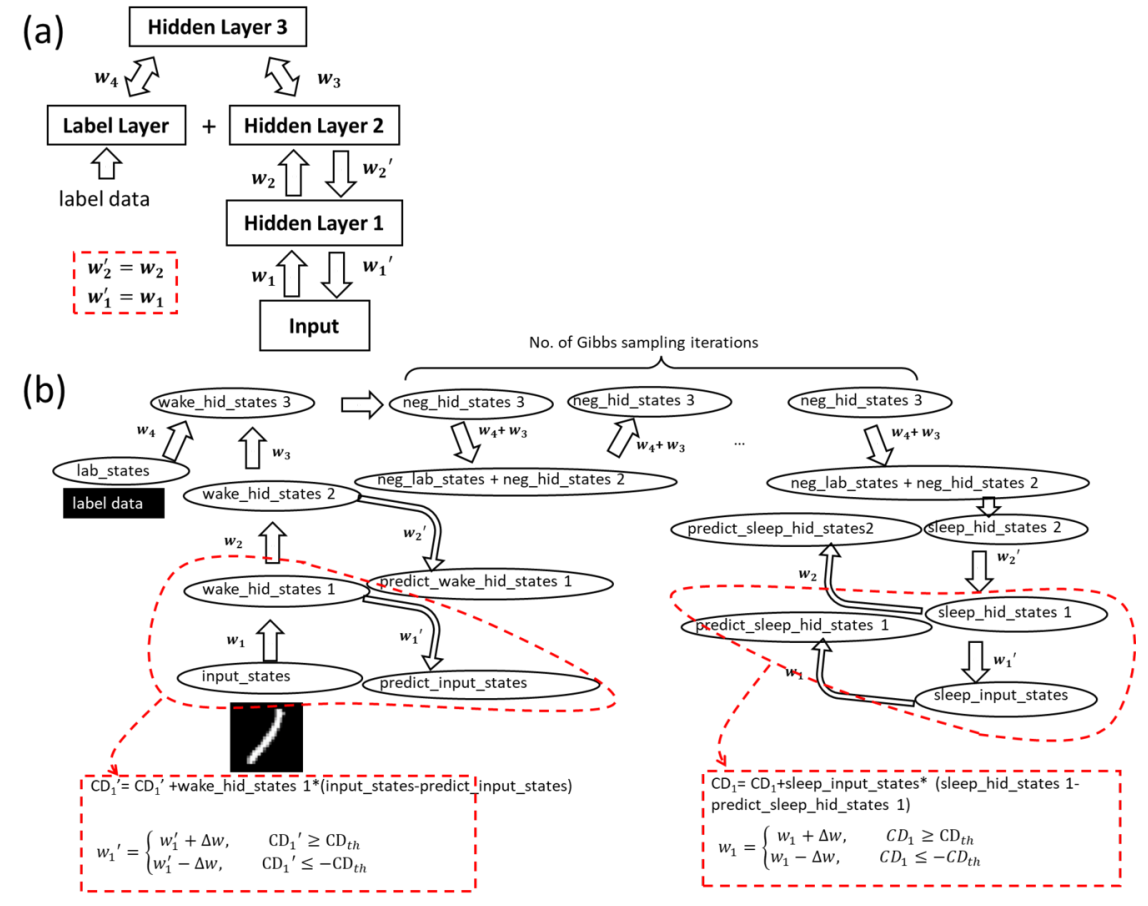

Figure S2. a) Deep belief network structure for the fine-tuning procedure by the wake-sleep algorithm. b) Procedure of utilizing wake-sleep algorithm for the fine tune of the deep belief net.

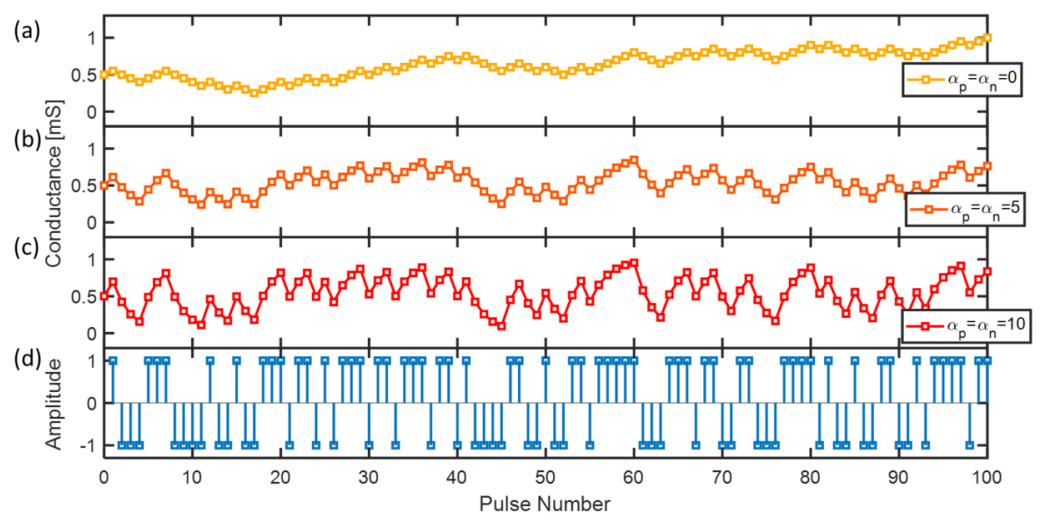

Figure S3. Example traces of the conductance updates as the responses of potentiation or depression voltage pulses. a) Weight update traces of an ideal device with linear weight update ability. b, c) Weight update responds of an ideal device with non-linear weight update behavior. d) Potentiation (positive) or depression (negative) pulse applied on the devices. 

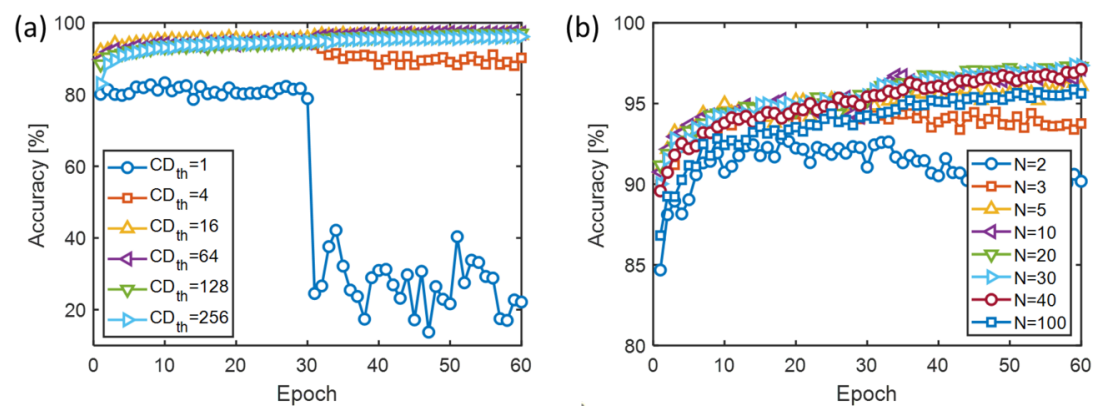

Figure S4. a) Test accuracy (50 times sampling) as a function of the training epoch for different CDth using the symmetric and linear weight update behavior. b) Test accuracy as a function of the training epoch for different numbers of conductance levels, $\mathrm{N}=\mathrm{N}_{\mathrm{p}}=\mathrm{N}_{\mathrm{d}}$.
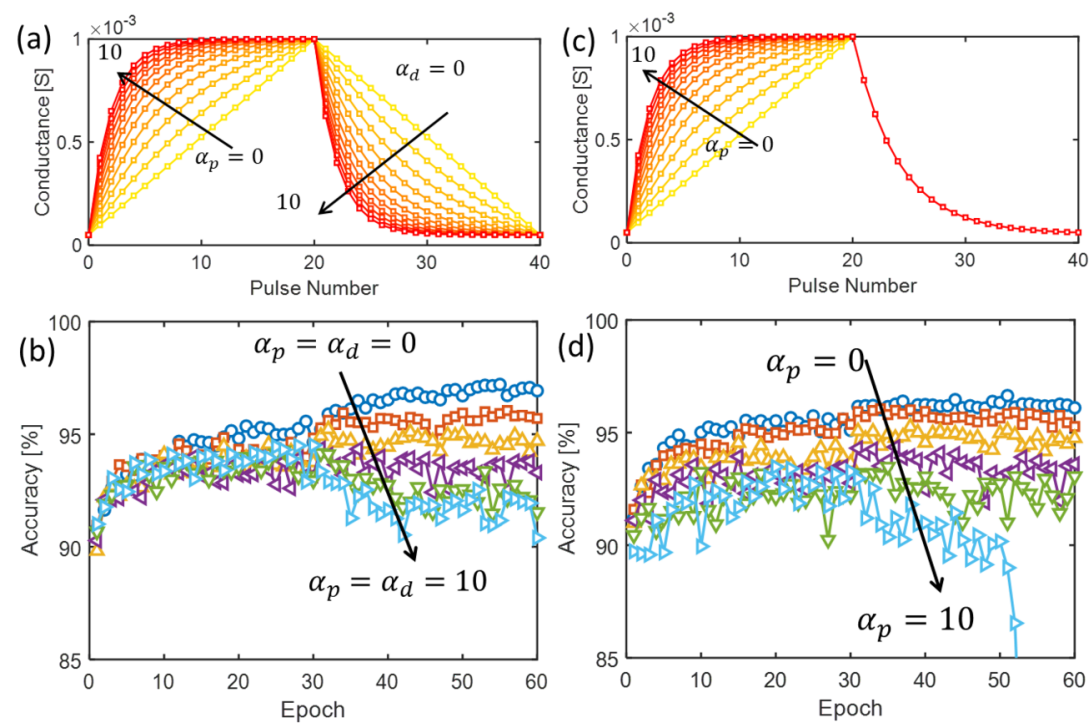

Figure S5. a) Modelled weight update behavior with different degrees of non-linearities (assuming the degrees of non-linearity in the potentiation and depression are the same). b) Test accuracy as a function of the training epoch for different degrees of symmetric nonlinearities of the weigh updates. c) Modelled weight update behavior with different degrees of non-linearities (assuming the degree of non-linearity in depression is constant, i.e., asymmetric weigh updates). d) Test accuracy as a function of the training epoch for different degrees of asymmetric nonlinearities of the weigh updates. 

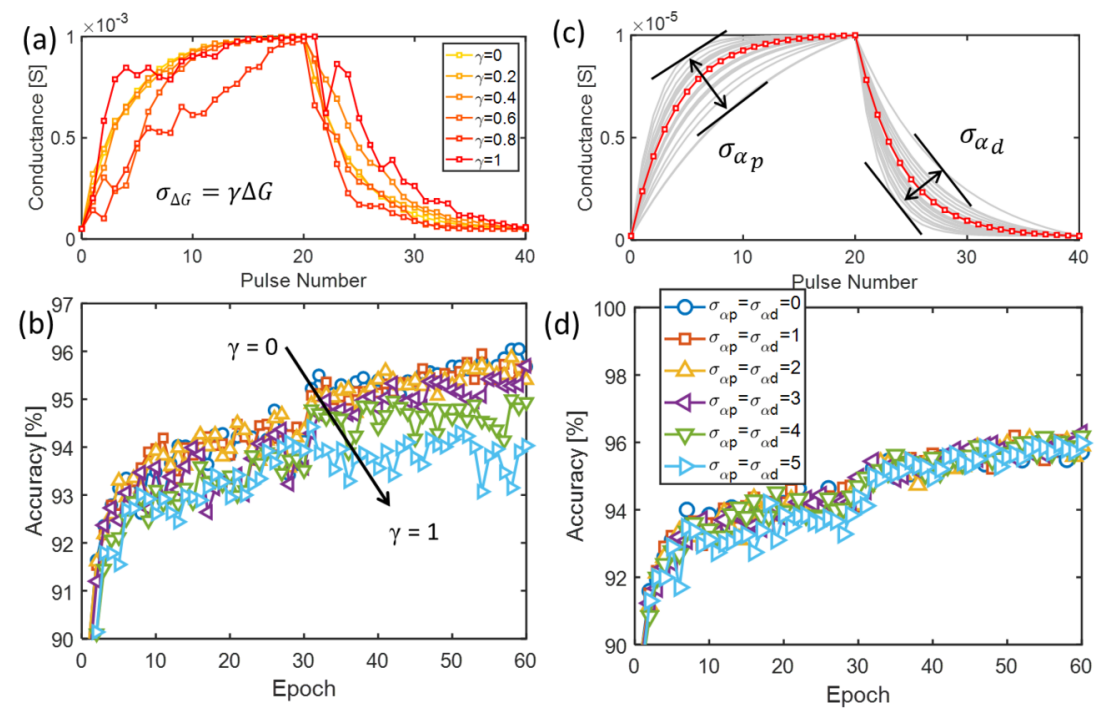

Figure S6. a) Modelled weight update behavior with different degrees of cycle-to-cycle variations. b) Test accuracy as a function of the training epoch for different degrees of cycle-to-cycle variations. c) Modelled weight update behavior with different degrees of device-to-device variations. d) Test accuracy as a function of the training epoch for different degrees of device-to-device variations.
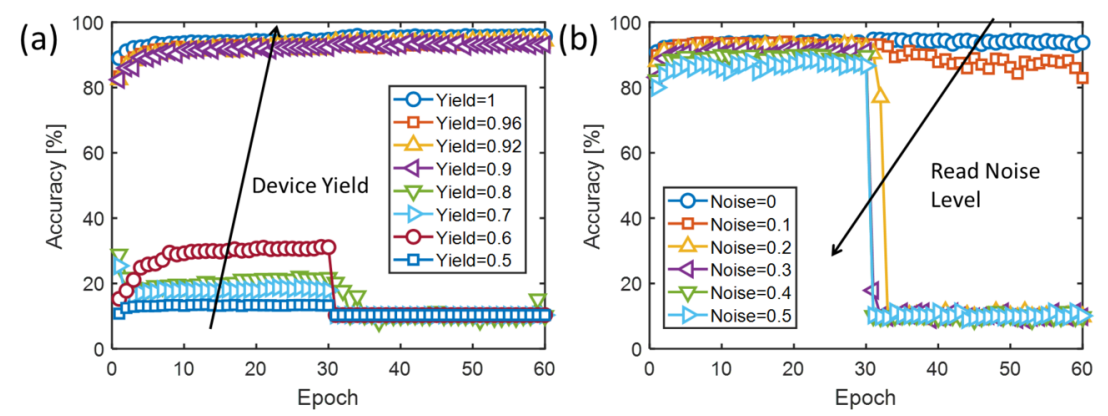

Figure S7. a) Test accuracy as a function of the training epoch for various yields of the memristive device in the array. b) Test accuracy as a function of the training epoch for various read noise levels of the memristive device.

(a)

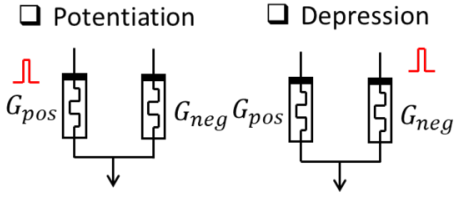

(b)

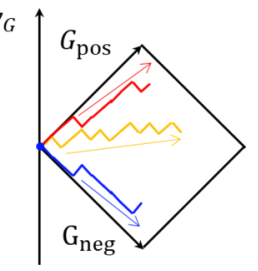

(c)

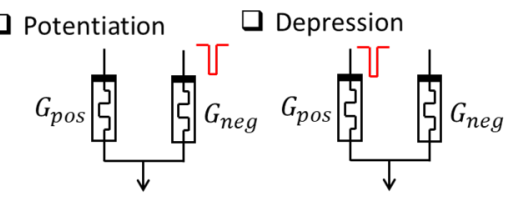

(d)

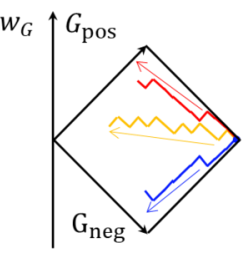


Figure S8. a) For the device that only has gradual conductance incremental behavior (PCM device in Fig. 5e), potentiation and depression operation are performed in the positive branch and negative branch of the synaptic cell consisting of a differential pair of the memristive devices, respectively. b) Illustration of typical synaptic evolution of the differential pair. The two memristive devices are initialized to low conductance status. Increment of the conductance of the positive branch results in the gradual increase of the overall synaptic weight. Vice-versa increment the conductance of the negative branch results in the gradual decrease of the overall synaptic weight. However, after multiple weight update operations, the differential pair might reach a status that both the devices are in high conductance states, which prevents further weight update operations and degrades the performance of the neural network. In the training case in this work, no such issues were detected, since the number of weight update operations on each memristive synaptic cell is highly reduced. c) For the device only has gradual conductance decrement behavior (OxRRAM device in Fig. 5d), potentiation and depression operation are performed in the negative branch and positive branch of the synaptic cell consisting of a differential pair of the memristive devices, respectively. b) Illustration of typical synaptic evolution of the differential pair.

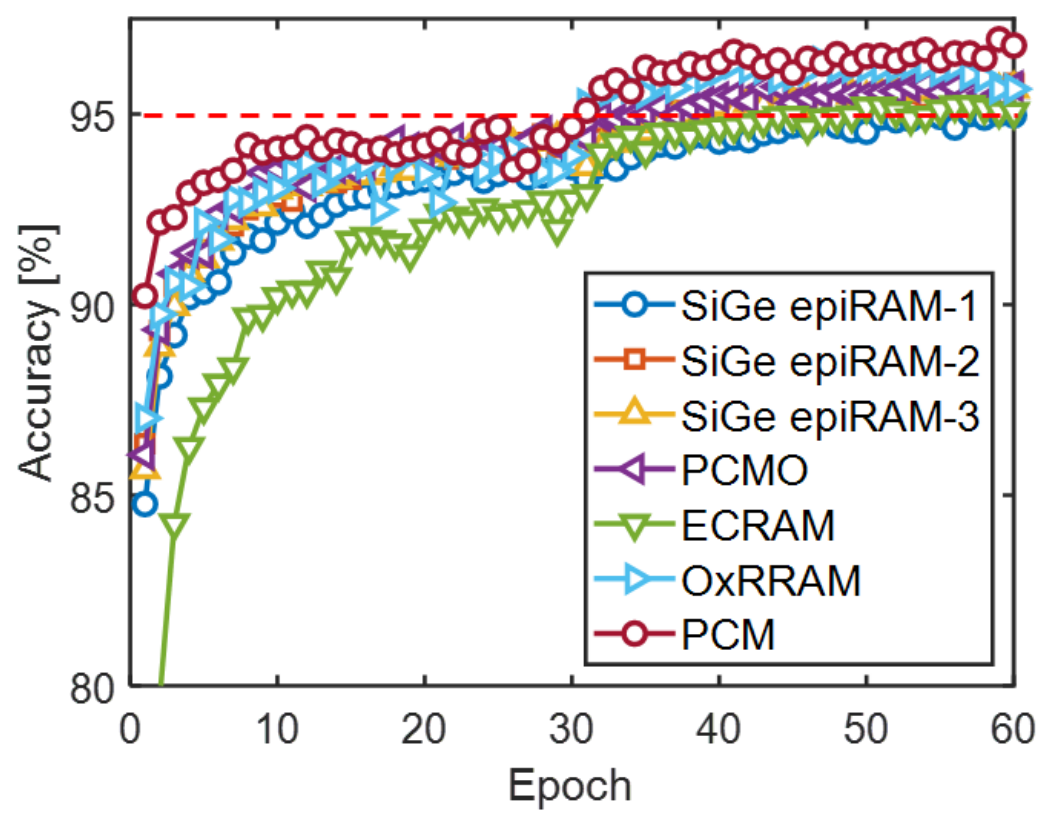

Figure S9. Test accuracy as a function of the training epoch when using various device data to train the neural network. 

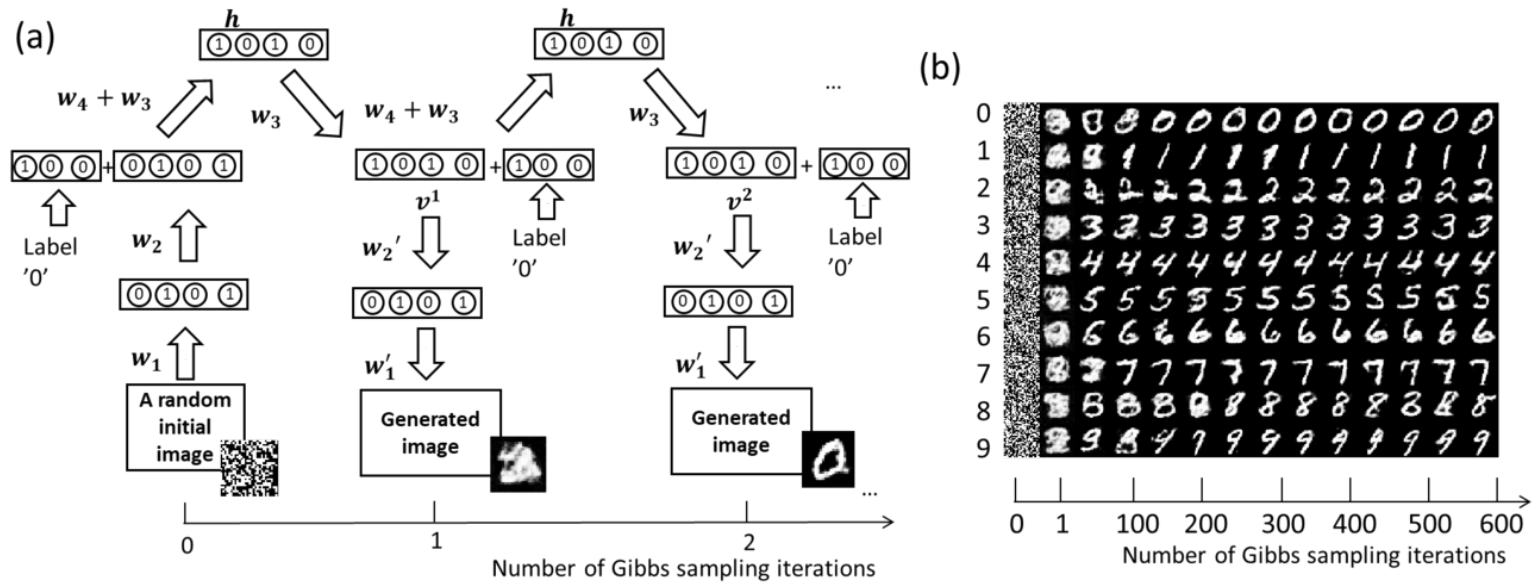

Figure S10. a) Schematic of using the trained DBN to generate handwritten digit images by only providing the labels. b) Generated handwritten digit images by providing only the labels.

Table S1. The fitting parameter values for the various memristive synaptic behaviors.

\begin{tabular}{lllllllll}
\hline Device & $G_{\max }$ & $G_{\min }$ & $N_{P}$ & $N_{d}$ & $a_{\pi}$ & $a_{\delta}$ & $\gamma$ & Accuracy \\
\hline SiGe epiRAM [9] & $40 \mu \mathrm{S}$ & $1 \mu \mathrm{S}$ & 500 & 400 & 8 & 15 & 2 & $95.01 \%$ \\
& $30 \mu \mathrm{S}$ & $0.1 \mu \mathrm{S}$ & 200 & 50 & 5 & 1 & 1 & $95.80 \%$ \\
& $12.5 \mu \mathrm{S}$ & $0.1 \mu \mathrm{S}$ & 100 & 50 & 1 & 1 & 1 & $95.76 \%$ \\
PCMO [10] & $0.16 \mu \mathrm{S}$ & $35 \mathrm{nS}$ & 100 & 100 & 6 & 20 & 1 & $95.78 \%$ \\
ECRAM [6] & $3 \mathrm{nS}$ & $1 \mathrm{nS}$ & 55 & 55 & 0.5 & 0.5 & 0.3 & $95.24 \%$ \\
OxRRAM [11] & $250 \mu \mathrm{S}$ & $20 \mu \mathrm{S}$ & - & 100 & - & 15 & 0.3 & $96.37 \%$ \\
PCM [12] & $2.2 \mathrm{mS}$ & $7 \mu \mathrm{S}$ & 30 & - & 6 & - & 0.3 & $96.96 \%$ \\
\hline
\end{tabular}

